\title{
CONF- $961110--4$ \\ NUCLEAR ASTROPHYSICS AT THE HOLIFIELD RADIOACTIVE ION BEAM FACILITY
}

\author{
Jeff C. Blackmon \\ Oak Ridge National Laboratory, PO Box 2008, Oak Ridge, TN 37831 \\ and \\ The University of North Carolina at Chapel Hill, Chapel Hill, NC 27599-3255
}

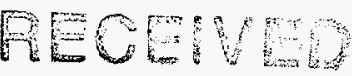

OCT 231903

OSTI

Reactions involving radioactive nuclei play an important role in explosive stellar events such as novae, supernovae, and $\mathrm{X}$-ray bursts. The development of accelerated, proton-rich radioactive ion beams provides a tool for directly studying many of the reactions that fuel explosive hydrogen burning. The experimental nuclear astrophysics program at the Holifield Radioactive Ion Beam Facility at Oak Ridge National Laboratory is centered on absolute cross section measurements of these reactions with radioactive ion beams. Beams of ${ }^{17} \mathrm{~F}$ and ${ }^{18} \mathrm{~F}$, important nuclei in the hot-CNO cycle, are currently under development at HRIBF. Progress in the production of intense radioactive fluorine beams is reported. The Daresbury Recoil Separator (DRS) has been installed at HRIBF as the primary experimental station for nuclear astrophysics experiments. The DRS will be used to measure reactions in inverse kinematics with the techniques of direct recoil detection, delayed-activity recoil detection, and recoil-gamma coincidence measurements. The first astrophysics experiments to be performed at HRIBF, and the application of the recoil separator in these measurements, are discussed.

\section{INTRODUCTION}

The rates of nuclear reactions are important parameters in many astrophysical models. In explosive stellar events, the temperatures and densities may be so extreme that reactions occur on time scales as short as seconds. Under such conditions, reactions involving radioactive nuclei play a key role in energy generation and nucleosynthesis. The rates of reactions involving radioactive nuclei are essential input for models of explosive events, but there exists little experimental information on these reactions (1). The primary objective of the nuclear astrophysics program at the Holifield Radioactive Ion Beam Facility (HRIBF) at Oak Ridge National Laboratory (ORNL) is the direct measurement of reactions involving radioactive nuclei that are important for astrophysics (2).

Radioactive ion beams are produced at HRIBF by an ISOL-type target/ion source (3-5). Intense light-ion beams from the Oak Ridge Isochronous Cyclotron (ORIC) pass through a high-temperature target. Reaction products diffuse out of the target material and pass through a transfer tube to an ion source, where they are ionized and extracted. The radioactive ion beam is then accelerated in the 25-MV tandem accelerator, so either a negative beam is extracted directly or a positive beam is extracted and charge exchanged. Two stages of mass analysis before injection into the tandem provide a mass resolution of 1 part in 20,000 .
Special attention must be given in an experiment with a radioactive ion beam to maximize the detection signalto-noise because of the relatively low beam intensities and high backgrounds. A versatile experimental station for astrophysics research is currently under construction at HRIBF centered around the Daresbury Recoil Separator. We discuss the installation of the separator and supporting equipment, and its planned application in the first experiments.

Most astrophysical reaction rates are dominated by contributions from low energy resonances. The spectroscopic properties of each resonance (resonance energy, spin, parity, total and partial widths) determine its contribution to the reaction rate (6). Beams produced by the ISOL technique are well-suited for the study of these resonance properties because intense beams can be produced at low energy with excellent mass and energy resolution. However, the target material must be carefully selected to maximize production of the species of interest, while at the same time allowing for fast diffusion of the reaction products out of the target. Likewise, the ion source must also be tailored to provide a high efficiency for the species of interest. A significant amount of development must be performed for each beam species (4).

Beams of radioactive fluorine are currently one focus of development at HRIBF. The ${ }^{17} \mathrm{~F}(\mathrm{p}, \gamma){ }^{18} \mathrm{Ne},{ }^{18} \mathrm{~F}(\mathrm{p}, \alpha){ }^{15} \mathrm{O}$, and ${ }^{18} \mathrm{~F}(\mathrm{p}, \gamma){ }^{19} \mathrm{Ne}$ reactions are all important in the hotCNO cycle, the sequence of reactions that fuel nova explosions (1). Two recent measurements using a
DISTRIBUTION OF THS DOCUMENT IS UNLMTER O
"The submitted manuscript has been authored by a contractor of the U.S. Government under contract No. DE-AC05-960R22464. Accordingly, the U.S. Government retains a nonexclusive, royalty-free license to publish or reproduce the published form of this contribution, or allow others to do so, for U.S. Government purposes." 


\section{DISCLAIMER}

This report was prepared as an account of work sponsored by an agency of the United States Government. Neither the United States Government nor any agency thereof, nor any of their employees, makes any warranty, express or implied, or assumes any legal liability or responsibility for the accuracy, completeness, or usefulness of any information, apparatus, product, or process disclosed, or represents that its use would not infringe privately owned rights. Reference herein to any specific commercial product, process, or service by trade name, trademark, manufacturer, or otherwise does not necessarily constitute or imply its endorsement, recommendation, or favoring by the United States Government or any agency thereof. The views and opinions of authors expressed herein do not necessarily state or reflect those of the United States Government or any agency thereof. 


\section{DISCLAIMER}

Portions of this document may be illegible in electronic image products. Images are produced from the best available original document. 
radioactive ${ }^{18} \mathrm{~F}$ beam have determined the strength of an important resonance in the ${ }^{18} \mathrm{~F}(\mathrm{p}, \alpha){ }^{15} \mathrm{O}$ reaction corresponding to a state at $E_{x}=7.063$ in the compound nucleus ${ }^{19} \mathrm{Ne}(7,8)$. These measurements have accurately determined the ${ }^{18} \mathrm{~F}(\mathrm{p}, \alpha){ }^{15} \mathrm{O}$ reaction rate for temperatures greater than $5 \times 10^{8} \mathrm{~K}$. The experimentally determined rate differs by as much as a factor of 10 from previous estimates based on incomplete spectroscopic information. These new results emphasize the need for direct measurements of important reaction rates using radioactive ion beams. The rates of the ${ }^{17} \mathrm{~F}(\mathrm{p}, \gamma){ }^{18} \mathrm{Ne}$ and ${ }^{18} \mathrm{~F}(\mathrm{p}, \gamma){ }^{19} \mathrm{Ne}$ reactions remain uncertain, and the spectroscopic properties of lower energy states in ${ }^{19} \mathrm{Ne}$ must be measured to accurately determine the ${ }^{18} \mathrm{~F}(\mathrm{p}, \alpha){ }^{15} \mathrm{O}$ reaction rate for $\mathrm{T}<5 \times 10^{8} \mathrm{~K}$, temperatures characteristic of most novae. We discuss recent progress that has been made at HRIBF in the development of radioactive fluorine beams, and initial measurements planned with these beams.

We are also planning a measurement of the ${ }^{7} \mathrm{Be}(\mathrm{p}, \gamma)^{8} \mathrm{~B}$ reaction. The next generation of neutrino detection experiments, which are coming online in the next few years, will provide greatly improved measurements of the solar neutrino energy spectrum. The uncertainties in the predicted solar neutrino fluxes need to be reduced for comparison to these experiments. The largest nuclear physics uncertainty in the flux of high-energy neutrinos comes from uncertainty in the ${ }^{7} \mathrm{Be}(\mathrm{p}, \gamma)^{8} \mathrm{~B}$ reaction rate (9). Measurements using a radioactive ${ }^{7} \mathrm{Be}$ target have established the energy dependence of the cross section, but there is some uncertainty regarding the overall normalization (10). Our plans for a measurement of the ${ }^{7} \mathrm{Be}(\mathrm{p}, \gamma)^{8} \mathrm{~B}$ cross section in inverse kinematics using a radioactive ${ }^{7} \mathrm{Be}$ beam are also discussed.

\section{THE DARESBURY RECOIL SEPARATOR}

An experimental station for astrophysics experiments is being constructed at HRIBF with the Daresbury Recoil Separator (DRS) (11) as its core. The DRS separates the recoiling reaction products from the primary beam in two 2 -m-long ExB velocity filters, and a $50^{\circ}$ dipole bending magnet provides a $q / \mathrm{m}$ focus. Three sets of quadrupoletriplet magnets focus the beam, and two sextupole magnets remove higher-order aberrations. The DRS was transferred to ORNL in the fall of 1994, and the physical installation of the separator is complete: all of the elements have been assembled, aligned and tested. Initial conditioning of the high voltage plates $( \pm 300 \mathrm{kV})$ is currently underway.

Recoil detection is advantageous for reactions measured in inverse kinematics because of the strong focusing of the recoil particles at forward angles. The DRS has an acceptance of $\pm 2.5^{\circ}$, allowing for $100 \%$ detection efficiency in ( $p, \gamma)$ reactions and about 10-20\% efficiency for $(p, \alpha)$ reactions. Two interchangeable target chambers have been constructed for the DRS at the University of North Carolina at Chapel Hill and are currently being installed. One large chamber is designed to hold an array of silicon surface barrier detectors based on the LouvainEdenburgh Detector Array (LEDA) design $(12,13)$. The LEDA detector subtends between $10-40^{\circ}$ or $140-170^{\circ}$ when placed at forward or backward angles respectively. By placing the LEDA detector at back angles, recoil-alpha coincidences can be measured with relatively high efficiency, e.g. $10 \%$ for the ${ }^{18} \mathrm{~F}(\mathrm{p}, \alpha){ }^{15} \mathrm{O}$ reaction. A second, smaller chamber has been constructed for use with external gamma-ray detectors, such as an array of $\mathrm{BaF}$ detectors. This chamber allows for gamma-detectors to be placed close to the target for maximum efficiency. The two chambers mount on the same aligning base, and can be interchanged with ease.

In capture reactions, particularly $(p, \gamma)$ reactions, the recoiling reaction products have nearly the same momentum as the beam particles, but their velocities differ significantly more. The DRS is well-suited for these measurements because of the two long velocity filters. For example, in the ${ }^{17} \mathrm{~F}(\mathrm{p}, \gamma)^{18} \mathrm{Ne}$ reaction, the momentum difference between the ${ }^{17} \mathrm{~F}$ and ${ }^{18} \mathrm{Ne}$ is less than $2 \%$, but the separation between the two will be greater than $2 \mathrm{~cm}$ at the midpoint between the two velocity filters. We have increased the distance between the two velocity filters slightly from the original configuration, and have installed a new set of slits between the two velocity filters. These new slits will allow rejection of a large fraction of the primary beam far upstream of the focal plane, and any particles that scatter from these slits will be removed from the beam in the second velocity filter. An ion optical solution to optimize the performance of the DRS has been developed. Based upon previous results using a recoil separator in inverse kinematics (14), we estimate primary beam rejection by the DRS to be 1 part in $10^{12}$.

The focal plane detector system developed for the DRS has recently been tested in stable beam measurements at Yale University. The system consists of the $\triangle \mathrm{E}-\mathrm{E}$ gas ionization counter used at Daresbury and two carbon-foil, microchannel plate detectors. The $\Delta \mathrm{E}-\mathrm{E}$ gas ionization counter allows for clear mass identification of the detected particles. The first microchannel plate detector gives position and timing information. The second microchannel detector gives timing information for timeof-flight measurements. Once the focal plane detector system is installed, commissioning of the DRS with stable beams will begin.

\section{${ }^{17} \mathrm{~F}$ AND ${ }^{18} \mathrm{~F}$ BEAMS}

Beams of ${ }^{18} \mathrm{~F}$ and ${ }^{17} \mathrm{~F}$ are currently being produced in tests at HRIBF using an electron beam plasma target/ion 


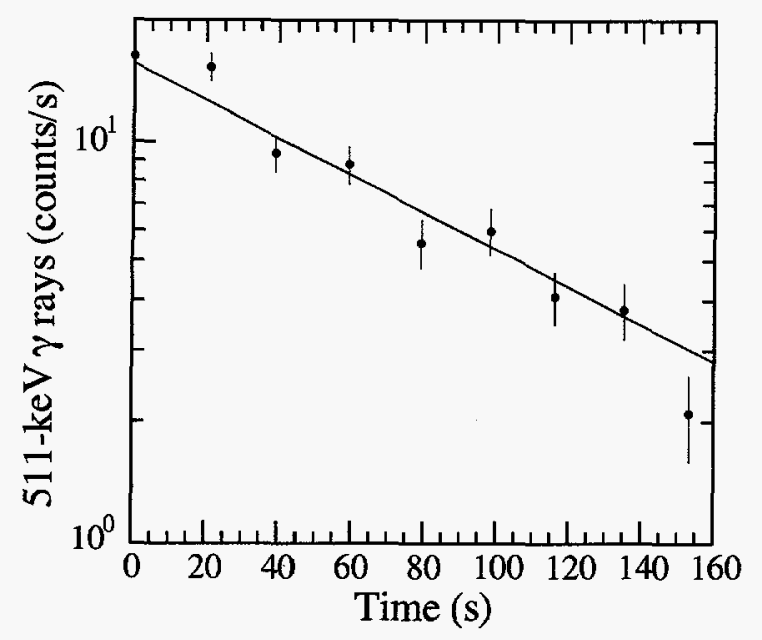

FIGURE 1. Measured activity following the implant of mass 44 ( $\left.\mathrm{Al}^{17} \mathrm{~F}\right)$. The line shows the expected decay from the $64.5 \mathrm{~s}$ half-life of

source and a fibrous $\mathrm{Al}_{2} \mathrm{O}_{3}$ target material. $\mathrm{A}$ high yield of either ${ }^{18} \mathrm{~F}$ or ${ }^{17} \mathrm{~F}$ is produced from the ${ }^{18} \mathrm{O}(\mathrm{p}, \mathrm{n})^{18} \mathrm{~F}$ or ${ }^{16} \mathrm{O}(\mathrm{d}, \mathrm{n}){ }^{17} \mathrm{~F}$ reactions on $\mathrm{Al}_{2} \mathrm{O}_{3}$. The fibrous material contains short path lengths for diffusion, and sustains high temperatures without sintering. Beams of positive ions are extracted from the ion source, mass analyzed using the UNISOR isotope separator, and implanted onto a movable tape. The radioactive fluorine current is then determined from the measured yield of $511-\mathrm{keV}$ gamma rays. The activity is measured as a function of time to confirm that the half-life of the activity corresponds to that of ${ }^{17} \mathrm{~F}$ or ${ }^{18} \mathrm{~F}$.

Because of the extreme reactivity of atomic fluorine, it is likely the fluorine isotopes are transported in molecular form. Most $(95 \%)$ of the ${ }^{17} \mathrm{~F}$ intensity we observe is at mass 44 , and most of the ${ }^{18} \mathrm{~F}$ at mass 45 . Figure 1 shows the measured activity of $511-\mathrm{keV}$ gamma rays following the implantation of mass 44 (with a deuteron driver beam). We conclude that fluorine which is released from the target promptly is released primarily as AlF. At a target temperature of $1500^{\circ} \mathrm{C}$, our preliminary measurements of the source efficiency are $2.4_{-0.5}^{+1.2} \times 10^{-5}$ for $\mathrm{Al}^{17} \mathrm{~F}$ and $6_{-1}^{+3} \times 10^{-4}$ for $\mathrm{Al}^{18} \mathrm{~F}$, with a Xe efficiency of $1.5 \%$. The uncertainties include estimates of the systematic uncertainties, which are much greater than the statistical uncertainties. The hold-up time in the source was measured for $\mathrm{Al}^{18} \mathrm{~F}$ to be $16.7 \pm 0.7 \mathrm{~m}$. This hold-up time is quite long compared to the $1: 25$ ratio of the $\mathrm{Al}^{17} \mathrm{~F}$ to $\mathrm{Al}^{18} \mathrm{~F}$ efficiencies, indicating that a small fraction of the $\mathrm{AlF}$ is released on a time scale much faster than $16.7 \mathrm{~m}$. We are currently investigating the effects of various operating parameters, such as target temperature, on the efficiencies and hold-up time. Further details of the fluorine beam development may be found in Stracener (15).

The ${ }^{17} \mathrm{~F}(\mathrm{p}, \gamma){ }^{18} \mathrm{Ne}$ reaction rate is believed to be dominated by an unconfirmed resonance corresponding to an excited state in ${ }^{18} \mathrm{Ne}$ at $\mathrm{E}_{\mathrm{x}}=4.561 \mathrm{MeV}$ (16). Comparison to the analog states in ${ }^{18} \mathrm{O}$ indicates that this state may be a $3^{+}$state, making it a strong s-wave resonance. We are planning a study of ${ }^{17} \mathrm{~F}(\mathrm{p}, \mathrm{p}){ }^{17} \mathrm{~F}$ scattering cross section in the energy region of the 4.561 $\mathrm{MeV}$ state using a radioactive ${ }^{17} \mathrm{~F}$ beam and a thin $(5$ $\left.\mu \mathrm{g} / \mathrm{cm}^{2}\right) \mathrm{CH}_{2}$ target. The scattered protons will be detected in the LEDA array. Given the present source efficiency for $\mathrm{Al}^{17} \mathrm{~F}$, a ${ }^{17} \mathrm{~F}$ beam current greater than $10^{5} \mathrm{~s}^{-1}$ on target can be achieved by accelerating AlF in the tandem accelerator, and dissociating it in the terminal. We will measure an excitation function (at 10-15 different energies). By summing the azimuthal and some radial segmentation of the LEDA array, less than 5\% statistics can be achieved in one day per energy with a beam current of $10^{5} \mathrm{~s}^{-1}$. If the presence of the resonance is confirmed, we will utilize the full radial segmentation of the LEDA array to measure the angular distribution of the scattered protons to determine the spin and parity of the resonance.

A significant increase in fluorine current should be obtainable with the current source design. For example, the overall (Xe) efficiency of the particular source used in these tests was about a factor of 6 less than that of other sources of the same design. We are also investigating design changes that could result in substantial improvement in the efficiency for AlF. An increase in the efficiency by a factor of a few hundred would result in sufficient beam intensity for measurements of the ${ }^{17} \mathrm{~F}(\mathrm{p}, \gamma){ }^{18} \mathrm{Ne}$ and ${ }^{18} \mathrm{~F}(\mathrm{p}, \gamma){ }^{19} \mathrm{Ne}$ reactions, as well as of the ${ }^{14} \mathrm{O}(\alpha, p){ }^{17} \mathrm{~F}$ reaction by measuring the inverse reaction ${ }^{17} \mathrm{~F}(\mathrm{p}, \alpha){ }^{14} \mathrm{O}$. The rate of the ${ }^{14} \mathrm{O}(\alpha, \mathrm{p}){ }^{17} \mathrm{~F}$ reaction is particularly important in determining the amount of "leakage" of material out of the hot-CNO cycle to higher masses (1).

\section{THE ${ }^{7} B E(p, \gamma)^{8}$ B REACTION}

Six measurements of the ${ }^{7} \mathrm{Be}(\mathrm{p}, \gamma)^{8} \mathrm{~B}$ reaction have been reported. The four most precise of these measurements differ in overall normalization by about $30 \%$. The difference is most likely due to systematic uncertainties, for example in the ${ }^{7} \mathrm{Be}$ target density. We are planning a measurement of the ${ }^{7} \mathrm{Be}(\mathrm{p}, \gamma)^{8} \mathrm{~B}$ cross section in inverse kinematics using a ${ }^{7} \mathrm{Be}$ beam. The ${ }^{7} \mathrm{Be}$ beam will be produced by a sputter ion source as $\mathrm{BeO}$, and dissociated in the terminal of the tandem. A second, high-energy stripper foil will produce a high efficiency of ${ }^{7} \mathrm{Be}^{4+}$, so that the tandem analyzing magnet can be used to help eliminate $\mathrm{Li}$ contaminants from the beam.

A thin $\mathrm{CH}_{2}$ target $\left(3 \times 10^{18}\right.$ atoms $\left./ \mathrm{cm}^{2}\right)$ will be used, and the cross section will be measured by detecting recoiling ${ }^{8} \mathrm{~B}$ nuclei in the Daresbury Recoil Separator. Tests of the $\triangle E-E$ gas ionization counter show it has sufficient resolution to distinguish ${ }^{7} \mathrm{Be}$ and ${ }^{8} \mathrm{~B}$. With a ${ }^{7} \mathrm{Be}$ 
beam current of $100 \mathrm{pA}$, we expect 55 counts/day at a center-of-mass energy of $1 \mathrm{MeV}$. Therefore, a run of 8 days duration is required to achieve $5 \%$ statistics. We expect 15 counts/day at a center-of-mass energy of $0.4 \mathrm{MeV}$. A somewhat longer run will be required at this lower energy, but a thicker target could reduce the required beam time. While this approach also has systematic difficulties, it is a different technique from that used previously. A similar experiment is currently being conducted by the Napoli-Bochum (NABONA) collaboration, but using a windowless hydrogen gas cell $(17,18)$.

\section{CONCLUSION}

The astrophysics endstation at HRIBF will allow for a high detection efficiency for a variety of techniques, such as direct recoil detection, delayed-activity recoil detection, and recoil-gamma and recoil-charged-particle coincidence measurements. Stable beam commissioning of the device will begin following installation of the target chamber and focal plane.

Our initial tests with fibrous $\mathrm{Al}_{2} \mathrm{O}_{3}$ as a target material for the production of ${ }^{17} \mathrm{~F}$ and ${ }^{18} \mathrm{~F}$ beams are encouraging. We have produced $\mathrm{a}^{17} \mathrm{~F}$ beam with intensity sufficient for measurement of ${ }^{17} \mathrm{~F}(\mathrm{p}, \mathrm{p}){ }^{17} \mathrm{~F}$ scattering cross sections. An improvement in efficiency by about a factor of 100-1000 would result in sufficient current for a measurement of the $17 \mathrm{~F}(\mathrm{p}, \mathrm{g}) 18 \mathrm{Ne}$ and $18 \mathrm{~F}(\mathrm{p}, \mathrm{g}) 19 \mathrm{Ne}$ reactions, as well as the ${ }^{14} \mathrm{O}(\alpha, \mathrm{p}){ }^{17} \mathrm{~F}$ reaction by a measurement of the inverse reaction, ${ }^{17} \mathrm{~F}(\mathrm{p}, \alpha){ }^{14} \mathrm{O}$ reaction. We are also developing a measurement of the ${ }^{7} \mathrm{Be}(\mathrm{p}, \gamma)^{8} \mathrm{~B}$ reaction using a radioactive ${ }^{7}$ Be beam.

\section{ACKNOWLEDGMENTS}

The development of radioactive fluorine beams is being conducted at ORNL by D. W. Bardayan, J. C. Blackmon, H. K. Carter, J. Kormicki, A. H. Poland, M. S. Smith, and D. W. Stracener. The Daresbury Recoil Separator is being installed by D. W. Bardayan, J. C. Blackmon, D. E. Pierce, and M. S. Smith. Oak Ridge National Laboratory is managed by Lockheed Martin Energy Research Corporation for the U.S. Department of Energy under Contract No. DE-AC05-960R22464.

\section{REFERENCES}

1. Champagne, A. E., and Wiescher, M., Annu. Rev. Nucl. Part. Sci. 42, 39-76 (1992).

2. Smith, M. S., Nucl. Inst. Meth. Phys. Res. B99, 349-353 (1995).

3. Garrett, J. D., Alton, G. D., Baktash, C., Olsen, D. K., and Toth, K. S., Nucl. Phys. A557, 701c-714c (1993).

4. Alton, G. D., Haynes, D. L., Mills, G. D., and Olsen, D. K., Nucl. Inst. Meth. Phys. Res. A328, 325-329 (1993).
5. Ravn, H. L., Nucl. Inst. Meth. Phys. Res. B70, 107-117 (1992).

6. Rolfs, C. E., and Rodney, W. S., Cauldrons in the Cosmos, Chicago: U. of Chicago Press, 1988.

7. Rehm, K. E., et al., Phys. Rev. C 53, 1950-1954 (1996)

8. Coszach, R., et al., Phys. Lett. B353, 184-188 (1995).

9. Bahcall, J. N., and Pinsonneault, M. H., Rev. Mod. Phys. 64, 885 (1992).

10. Filippone, B. W., Ann. Rev. Nucl. Part. Sci. 36, 717 (1986).

11. James, A. N., Morrison, T. P., Ying, K. L., Connell, K. A., Price, H. G., and Simpson, J., Nucl. Inst. Meth. Phys. Res. A267, 144152 (1988).

12. Bain, C., Ph. D. Dissertation, U. of Edinburgh, 1996, pp. vi-xxix.

13. Sellin, P. J., et al., Nucl. Inst. Merh. Phys. Res. A311, 217-223 (1992).

14. Smith, M. S., Rolfs, C. E., and Barnes, C. A., Nucl. Inst. Meth. Phys. Res. A306, 233- (1991).

15. Stracener, D. W., Carter, H. K., Kormicki, J., Poland, A. H., Smith, M. S., Blackmon, J. C., Bardayan, D. W., "Studies on the Production and Release of $F$ and As Isotopes from the HRIBF Target/Ion Source," in Proceedings of the 14th International Conference on the Application of Accelerators in Research and Industry, 1996.

16. Garcia, A., Adelberger, E. G., Magnus, P. V., Markoff, D. M. Swartz, K. B., Smith, M. S., Hahn, K. I., Bateman, N., and Parker, P. D., Phys. Rev. C 43, $2012-2019$ (1991).

17. Gialanella, L., et al., preprint, accepted for publication in $\mathrm{Nucl}$. Inst. Meth. Phys. Res. A.

18. Campajola, L., et al., preprint, accepted for publication in Zeit. fur Phys. 